Research Article

\title{
Determining the quality of the powders of Xanthium Strumarium and Xanthium Spinosum by microscopic examination and preliminary tests
}

\author{
Mohammad Ghannam ${ }^{1} \cdot$ Esam Shammaa $^{1} \cdot$ Adnan Ali-Nizam $^{2}$
}

Received: 6 May 2020 / Accepted: 20 August 2020 / Published online: 31 August 2020

(c) Springer Nature Switzerland AG 2020

\begin{abstract}
Xanthium strumarium and Xanthium spinosum are found in many parts of the world and they are used in traditional medicine in many countries. Several studies have shown some medicinal effects for $X$. strumarium parts (leaves-seedlings-flowers-roots-fruits), and of the leaves of $X$. spinosum to treat some diseases. This paper is designed to set standards in determining the quality of the powders of these two plants. The standards include microscopic examinations and preliminary tests, which can be considered as identification tests for the purity of the powders. Contamination can occur during the collection of the plant, such as the presence of fruit elements among the powder of the leaves. Additionally, toxic substances in the fruits of the plant could be present that need special treatment before using them. Furthermore, these species of Xanthium are invasive to fields, so microscopic examination revealed the contamination of other plant powders with parts of these Xanthium species. The microscopic examination of $X$. strumarium leaves showed a distinctive nonglandular trichomes different from the ones in X. spinosum leaves. Seedling powder gives more glandular trichomes than the leaf powder, and the flower powder can be distinguished by the presence of distinctive pollen grains. The root powder is characterized by the presence of stone cells, while the presence of fibers indicates the elements of stems or fruits, depending on the shape of the fibers. The preliminary tests showed that all parts of $X$. strumarium contain alkaloids, but not in the leaves of $X$. spinosum. The type of flavonoids was different between the leaves of Xanthium species. The two species did not contain anthraquinones or saponins, while the leaves of $X$. spinosum contained tannins.
\end{abstract}

Mohammad Ghannam, mohammadghannam84@gmail.com | 'Department of Pharmacognosy, Faculty of Pharmacy, Damascus University, Damascus, Syria. ${ }^{2}$ Department of Plant Biology, Faculty of Science, Damascus University, Damascus, Syria. 


\section{Graphic abstract}

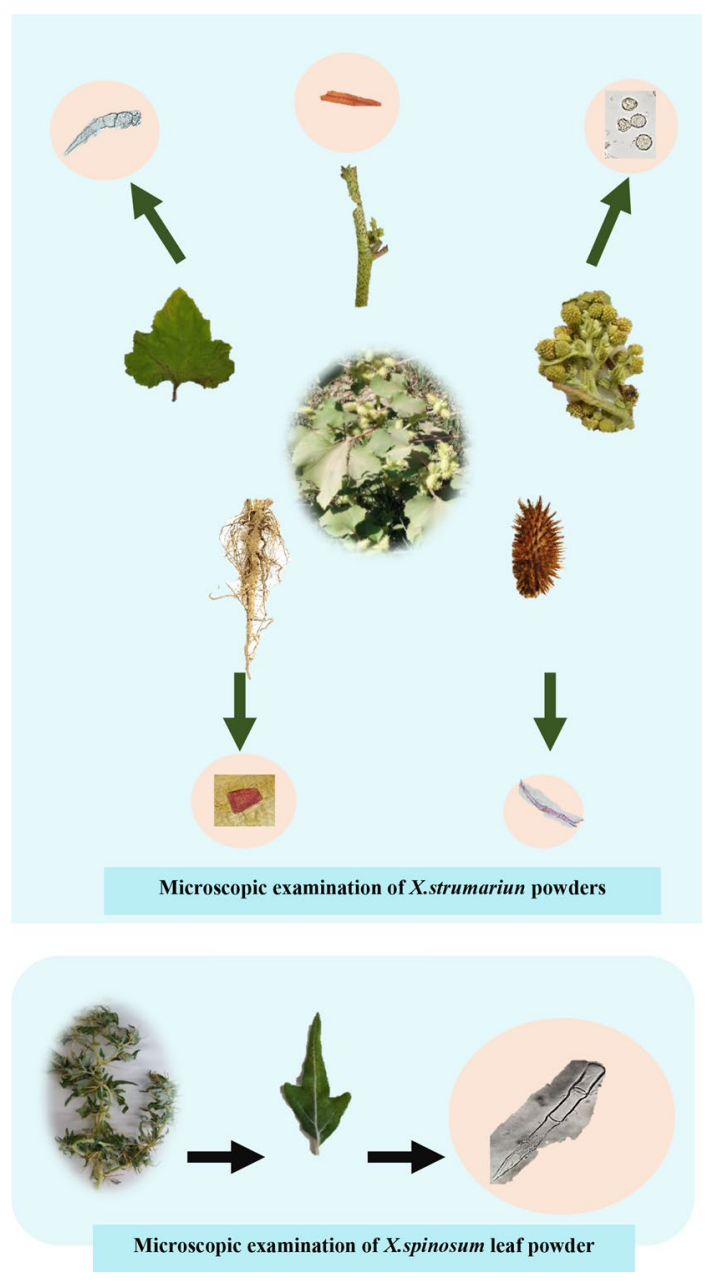

Keywords Xanthium strumarium · Xanthium spinosum · Microscopic examination · Quality

\section{Introduction}

Xanthium belongs to the Asteraceae family (formerly Compositae). There are more than 20 species of Xanthium genus in North America that can be distinguished based on the morphological characteristics of the burs [18], but Love and Dansereau [10] distinguished the genus with two distinctly different species: Xanthium strumarium and Xanthium spinosum. The spinosum is relatively stable, while the strumarium changes greatly and is divided into subspecies. Mouterde [12] mentions in Syrian and Lebanese flora that there are three species of this genus in Syria: $X$. strumarium, $X$. echinatum, $X$. spinosum, but the website of The Plant List. org has considered that $X$. echinatum is the same species as X. strumarium, and so there are truly only two unique main species in Syria.

According to Al-Hakim et al. [1] the morphology of $X$. strumarium is an annual herb length of $30-100 \mathrm{~cm}$ (Fig. 11), with leaves that are simple, alternating, without spines, triangular in general shape. The edges of the leaves are irregularly toothed and lobed. Flowers are unisexual, some inflorescences carry only male flowers, while others carry female flowers on the same plant, meaning that the plant is unisexual and monoecious. Flowers occur in heads clustered in axils. Male heads are spherical, tubular flowers, and are located at the ends of branches. Female heads are positioned below male heads, and every head includes two florets. The floret contains only the pistil without 
corolla and calyx, and the whole two florets enclosed within a spiny involucre. Fruit contains two achenes, surrounded by hard involucre that ending in two hooked beaks, and covered with hooked spines.

According to Al-Hakim et al. [1] also, the morphology of $X$. spinosum is: Annual herb, length $30-60 \mathrm{~cm}$ (Fig. 12). Leaves: contains three irregular lobes, each of which has a clear main vein in the middle. The upper surface of the leaves is dark green, and the lower surface of the leaves is paler than the upper surface. Each leaf has three branches of yellow spines, length $0.7-2.5 \mathrm{~cm}$. Fruits are similar to those of the first species, and this plant is distinguished from the other species by its relatively small in size fruits.

Although chemical methods of analysis, especially chromatography, are now accepted as standard techniques for the identification of many vegetal materials, microscopic structure has long been established as providing a most useful and reliable criterion [2].

This research aims to identify the microscopic elements of $X$. strumarium parts powders and $X$. spinosum leaf powder, so that their quality and purity are set by these standards. Seedlings and flowers of $X$. strumarium have not been studied microscopically before. Lastly, to determine the type of chemical components by preliminary tests to help differentiate the powder of the two species.

\section{Materials and methods}

\subsection{Plant samples and preparation of powders}

The seedlings (Se) of $X$. strumarium were collected in August 2017 from the Al-Bahloulia area east of Lattakia, Syria. Then the mature plants were collected from the same region. Fruits were obtained in November of the same year. As for the flowers, they were collected in September 2018. The second species $X$. spinosum was collected at the end of August 2017 from the Al-Husayn area, north of Tartous, and from the Wadi Al-Nadara area, west of Homs. The samples were dried in the shade for 14 days in a well-ventilated room.

The parts of $X$. strumarium were separated: leaves (Le)-stems (St)-roots (Ro)-fruits (Fr)-flower buds (Flo). $X$. spinosum leaves (Spi, the abbreviation of spinosum) were separated from the stem and spines. Plants samples were weighed after drying: Le: 500 g, St: 300 g, Ro: 175 g, Fr: 600 g, Flo: 150 g, Spi: $100 \mathrm{~g}$.

All parts were ground with an electric mill to obtain fine powders, which were then kept in tightly sealed glass containers, wrapped in dark bags so as not to be exposed to air and light, and stored away from sources of heat and moisture until the time of use.

\subsection{Microscopic examination}

Two types of solution were used for microscopy:

Chloral hydrate and glycerin: Chloral hydrate dissolves starch, proteins, chlorophyll, resins, and volatile oils, and causes shrunken cells to expand. It does not dissolve calcium oxalate and is therefore a good reagent for the detection of these crystals. Glycerin is useful for preparations that may be left for some time [7].

Phloroglucinol and hydrochloric acid: The powder was moistened with an alcoholic phloroglycinol solution and allowed for $2 \mathrm{~min}$ to dry, then a concentrated solution of hydrochloric acid was added. All lignified walls would stain pink or red [7].

With each solution; microscopic examination was done for three samples of each powder, and several photomicrographs were taken for each element. The best and clearest photomicrographs were selected for the microscopic measurement.

\subsection{Microscopic measurement}

It was accomplished by using an eyepiece micrometer and calibrating it with a precisely measured stage micrometer [17]. After the calibration, it was determined that one unit on the eyepiece micrometer equals approximately $9 \mu \mathrm{m}$ on the stage micrometer with the objective $10 x$, and $2.35 \mu \mathrm{m}$ with the objective $40 \times$.

\subsection{Preliminary tests}

Preliminary tests were conducted for the powders. The following components were detected and categorized: alkaloids-flavonoids-anthraquinones-saponins-tannins by the procedures mentioned in the Munajjed and Agha [13] manual.

\subsubsection{Detection of alkaloids}

By precipitation reactions:

A small amount of powder was boiled with a quantity of Hydrochloric acid $2 \mathrm{~N}+$ Water, then was detected from the filtrate with Dragendorff, Mayer, Wagner reagents.

\subsubsection{Detection of flavonoids}

Powder was extracted with methanol, and by adding the petroleum ether the unwanted matters were separated. The methanolic extract was used for detection in the following tests: 


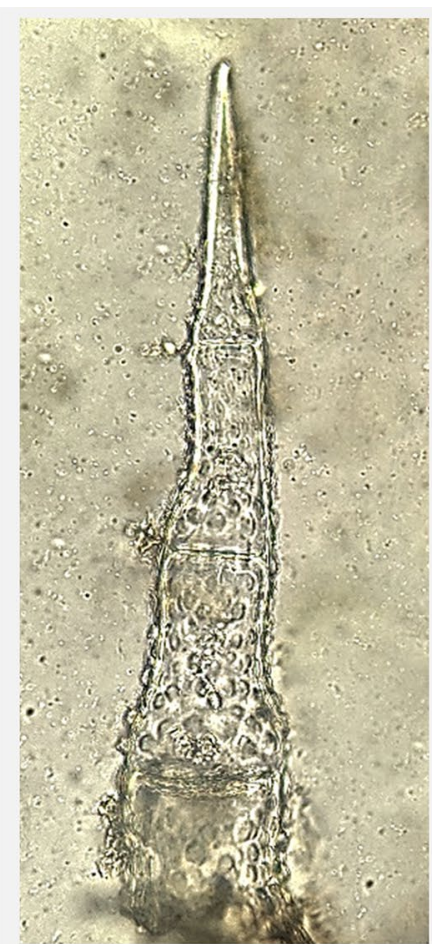

(a)

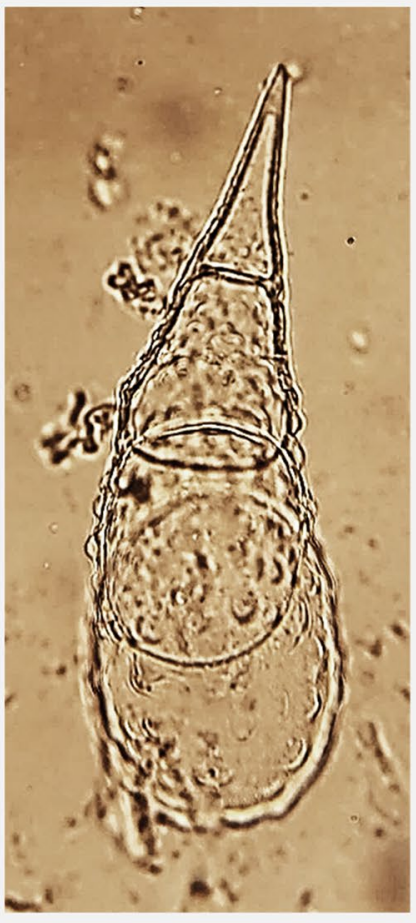

(b)

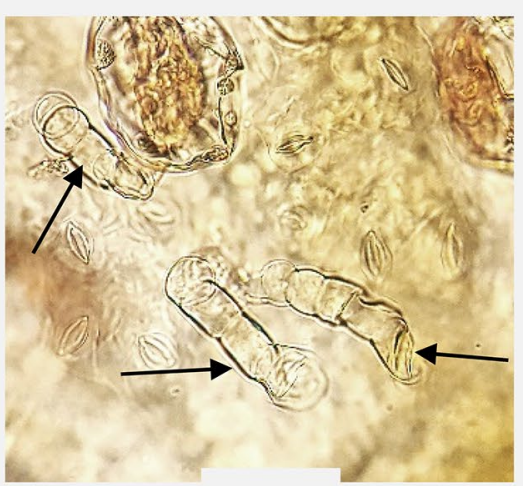

(c)

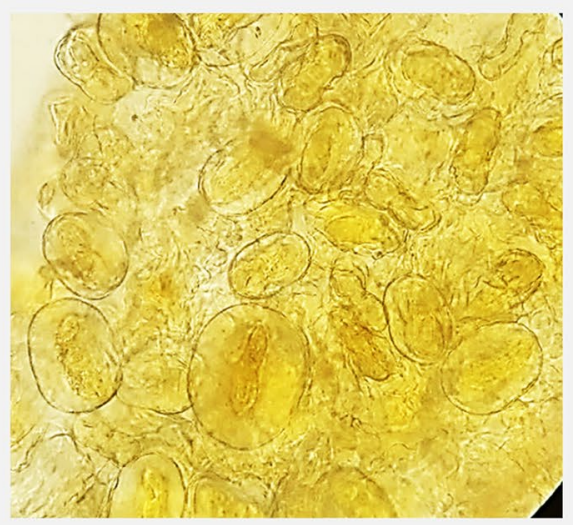

(d)

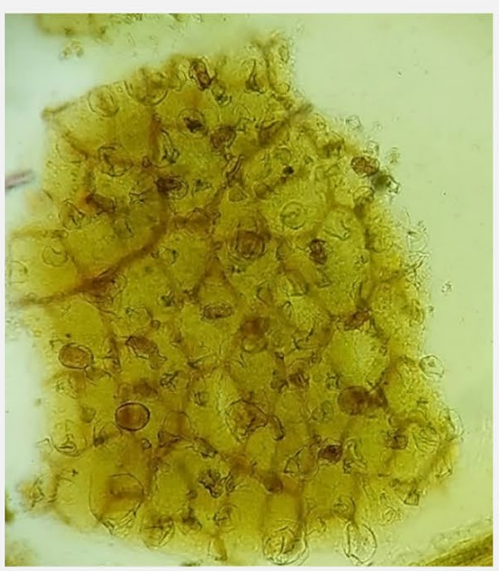

(f)

Fig. 1 Microscopic characteristics of X.strumarium leaves powder, a-c Non glandular trichomes. d Glandular trichomes. e Anomocytic stoma. f Mesophyll, $10 \times$

Wilson Taubock test: Methanolic extract was mixed with boric acid and oxalic acid. Formation of a fluorescent solution indicates the presence of flavonoids.

Aluminum chloride test: Methanolic extract was mixed with aluminum chloride. Then a drop of the resulting solution is placed on a filter paper and a UV fluorescent was observed. 


\subsubsection{Detection of tannins}

The powder was extracted with ethanol and the tannin was detected with ferric chloride solution. Formation of blue or green color transforming quickly to black indicates the presence of tannins.

\subsubsection{Detection of anthraquinones}

By boiling the powder with a quantity of Hydrochloric acid $7 \%$, extracting by ether, separating the ethylic ether layer, and adding to the ethereal solution about one-third of its volume of Ammonia. After shaking a pink color formation in the aqueous layer indicates the presence of Anthraquinones.

\subsubsection{Detection of saponins}

Foam Test: the powder was shaken with hot water. Foam formation persisting for $10 \mathrm{~min}$ indicates the presence of saponins.

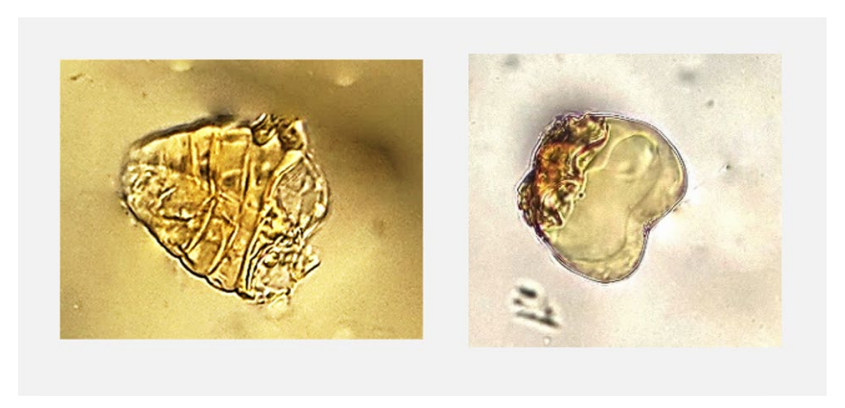

\section{Results}

\subsection{Microscopic examination of $X$. strumariun parts powders}

\subsubsection{Microscopic characteristics of the leaf powder}

- Nonglandular trichomes with three types:

A. Multicellular, uniseriate, with a sharp, spiny end, and clear protuberances. The total length did not exceed $30 \mu \mathrm{m}$ (Fig 1a).

B. Multicellular, uniseriate, with very sharp, spiny end, clear protuberances, and broader cells, approximately 15-20 $\mu \mathrm{m}$ in length (Fig. 1b). Sometimes these trichomes appear smaller 7-9 $\mu \mathrm{m}$ (only 2 cells).

C. Multicellular, uniseriate, with a smooth wall (Fig 1C).

- Glandular trichomes: Asteraceae family pattern: multicellular, biseriate head, and unicellular base. They were found in large numbers positioned on the surface of the epidermis that attached to mesophyll tissue (Fig 1d).

- Fragments of epidermis with Anomocytic stomata (Fig 1e).

- Fragments of mesophyll in surface view (Fig. 1f).

\subsubsection{Microscopic characteristics of the seedling powder}

The previous nonglandular trichomes of the mature leaves appear the same, in addition to the presence of:

Glandular trichomes (Asteraceae family pattern) appear free from epidermal tissue. This distinguishes seedling powder from mature leaf powder (Fig 2)

Fig. 2 Glandular trichomes of X.strumarium seedling powder

Fig. 3 Microscopic characteristics of $X$.strumarium roots powder. a Pitted vessels. b Stone cells

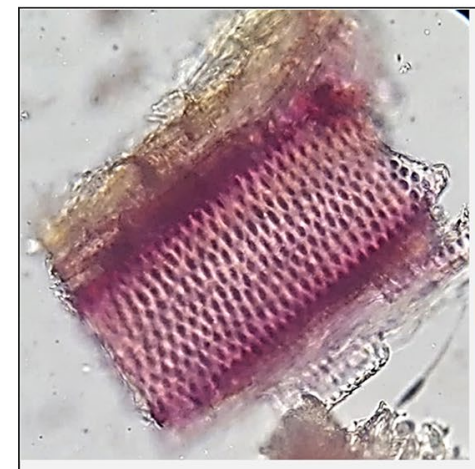

(a)

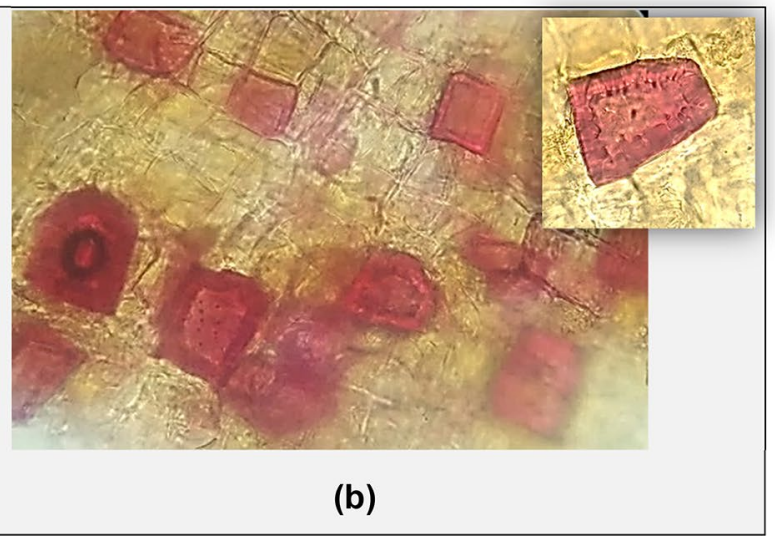


Fig. 4 Microscopic characteristics of X.strumarium flowers powder. a Pollen grains. $\mathbf{b}$ Endothecium. c Anther, $10 \times$ $40 \times$. d Epidermis of corolla

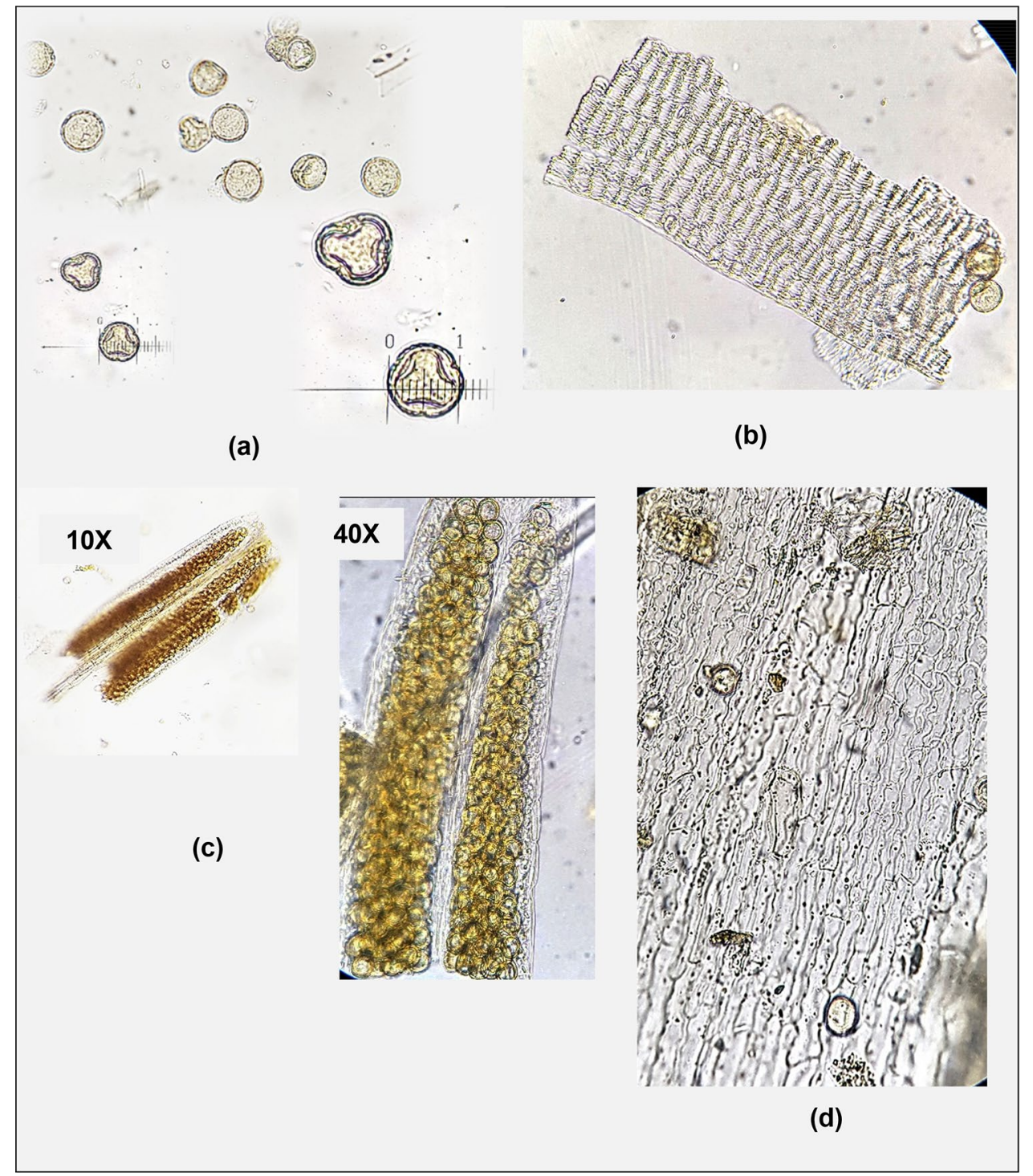

\subsubsection{Microscopic characteristics of the root powder}

After staining with phloroglucinol and hydrochloric acid, the following can be observed:

- Large fragments of pitted vessels, (Fig. 3a).

- Fragments of cortex containing scattered quadrate stone cells (Fig 3b).

\subsubsection{Microscopic characteristics of the flower powder}

- Spherical pollen grains, with a smooth exine, and some grains appear tricolporate, approximately $25-27 \mu \mathrm{m}$ in diameter (Fig 4a).

- Fragments of endothecium of the anther (surface view) (Fig 4b).

- Fragments of the whole anther (Fig 4c).
- Epidermis of floret corolla showing cells with wavy walls (Fig 4d).

\subsubsection{Microscopic characteristics of the fruit powder}

After staining with phloroglucinol and hydrochloric acid, the following can be observed:

- Fragments of the mesocarp attached to the endocarp (Fig. 5a, b).

- Fusiform fibers, approximately $35-45 \mu \mathrm{m}$ in length (Fig. 5c, d).

\subsubsection{Microscopic characteristics of stem powder}

After staining with phloroglucinol and hydrochloric acid, the following can be observed: 


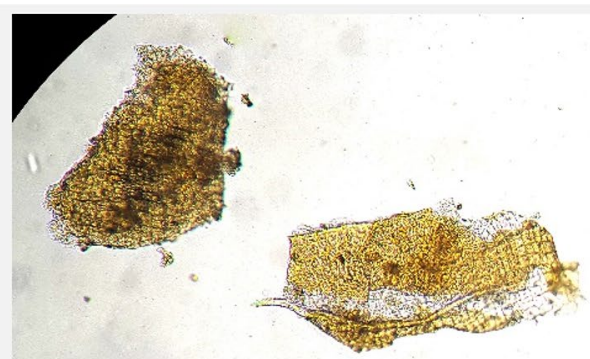

(a)

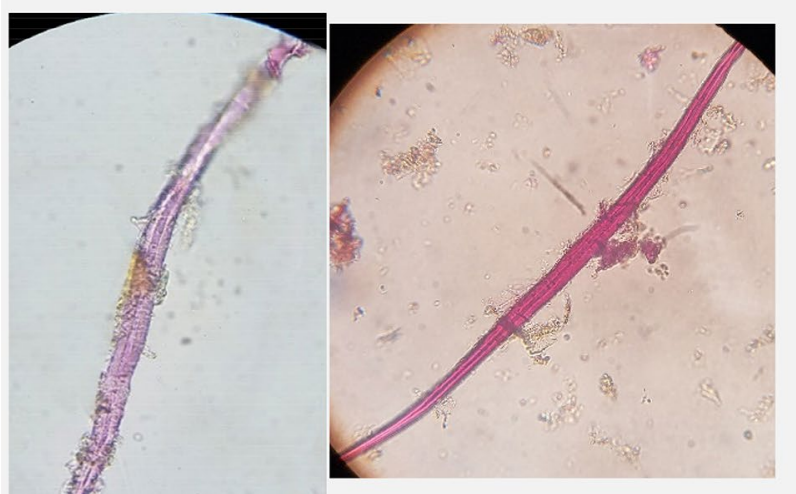

(c)

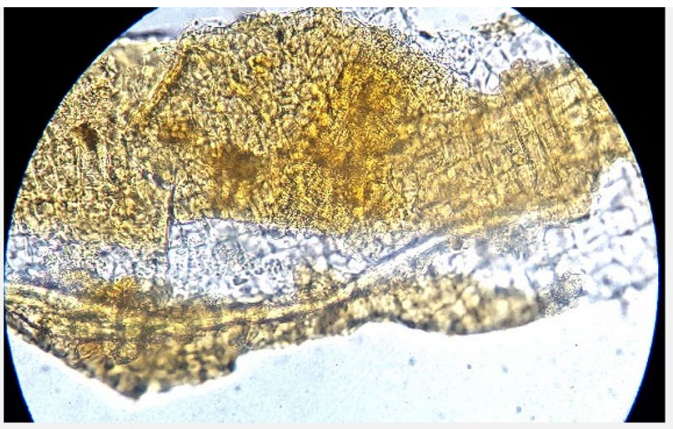

(b)

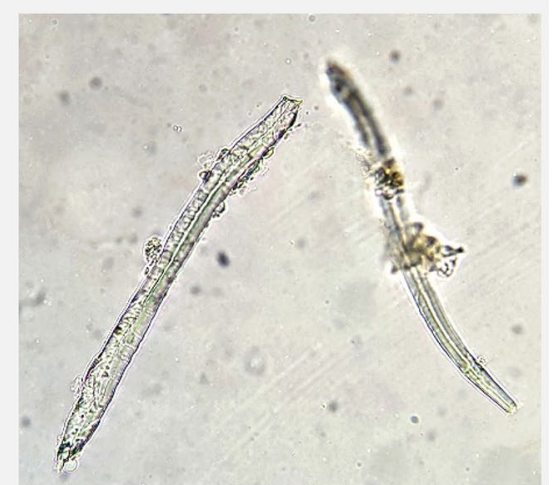

(d)

Fig. 5 Microscopic characteristics of X.strumarium fruits powder. a Endocarp, 10×. b Endocarp, $40 \times$. c Fiber, with staining. d Fiber, without staining

- Fibers, which have a dentate wall, and marked with pits lumen (Fig. 6a).

- Vessels with two types: pitted and spiral (Fig. 6b).

- Parenchyma cell contains rosette crystals (Fig. 6c).

- Fragments of polygonal cells contain purple pigment. These pigments are spots that appear on the surface of the stem (Fig. 6d)

\subsection{Microscopic characteristics of $X$. spinosum leaf powders}

- Fragments of mesophyll attached with epidermis, and numerous trichomes positioned on its surface (Fig. 7a).

- Nonglandular, multicellular, uniseriate trichomes. Their surface is smooth, approximately $30-40 \mu \mathrm{m}$ in length, and some trichomes appear 15-20 $\mu \mathrm{m}$ (Fig. 7b).

- Glandular trichomes (Asteraceae family pattern).

- Fibers indicate the presence of ground spiny with leaves (Fig. 7c).

\subsection{Microscopic distinction between $X$. strumariun leaf powder and $X$. spinosum leaf powder}

It can be distinguished by nonglandular trichomes, as in Table 1.

\subsection{Results of the preliminary tests}

- Alkaloids detection reactions: The following results were shown in Table 2.

- Flavonoids detection reactions: The following results were shown the intensity of UV fluorescence of Wilson Taubock tests, and the color of UV fluorescence of aluminum chloride test (Table 3, Fig. 8).

- Anthraquinones and saponins detection results were negative in all the plant parts, but tannins detection results were positive only with fruits and the leaves of $X$. spinosum (Table 4). 
Table 1 Distinguish between the nonglandular trichomes of the two species

X.strumarium
$\begin{aligned} & \text { Clear protuberances on the surface } \\ & \text { The lower cells are wider than the upper ones }\end{aligned}$

Table 2 Detection of alkaloids

\begin{tabular}{llll}
\hline $\begin{array}{l}\text { Reagent } \\
\text { Plant part }\end{array}$ & Dragendorff & Mayer & Wagner \\
\hline Le & + & - & \\
Se & + & - & + \\
Fr & + & + & + \\
Ro & - & - & - \\
Spi & - & - & + \\
\hline
\end{tabular}

(+) indicates precipitation

$(-)$ indicates no precipitation

Table 3 Detection of Flavonoids

\begin{tabular}{llllll}
\hline Plant part & Le & Se & Fr & Ro & Spi \\
\hline Wilson Taubock tests & ++ & + & - & - & ++ \\
Aluminum chloride test & Brown & Brown & Green & - & Green \\
\hline
\end{tabular}

$(++)$ indicates strong fluorescence

$(+)$ indicates moderate fluorescence

$(-)$ indicates no fluorescence

\section{Discussion}

In traditional medicine, the leaves, fruits, and roots are the parts that were used from the first species $X$. strumarium [9]. Many effectiveness studies were conducted on these parts, as well as for the leaves of the second species $X$. spinosum. Therefore, it is necessary to set standards to control the quality of the powders of these parts, and this research is a complement to previous research conducted on them. The microscopic examination of each part of $X$. strumarium, and $X$. spinosum leaves and the preliminary tests conducted in this study are useful in determining the following standards: the stage at which the plant was collected, detecting powder contamination during plant collection or grinding, determining of Xanthium species, and the type of chemical components contained in the two species.

$X$. strumarium leaves are the most used for medicinal purposes, and the microscopic characteristics were three types of multicellular nonglandular trichomes (Fig. 1a-c). Bhogaonkar and Ahmad [3], who provided illustrationswithout photomicrography-for the trichomes, indicated to the presence of a fourth type: nonglandular multicellular with multicellular base, but Fig. 9 shows that this type is the same one in Fig. 1, whereas the base of the trichome may appear and may not. Bhogaonkar and Ahmad [3] also indicated that there was a second type of glandular trichome, which was a scales type, but this type did not appear in our study. A possible proposed explanation for their observations is that it was a broken part of the nonglandular trichome or basal region of a trichome on a leaf, as seen in Fig. 10. Sravani and Lakshmi [15] mentioned that the type of stomata was anomocytic and sometimes anisocytic may appear as well as Bhogaonkar and Ahmad [3] observed. While Reeta et al. [14] findings the stomata were anisocytic. However, only the anomocytic type was noticed in our study (Figs. 1e, 11, 12). 


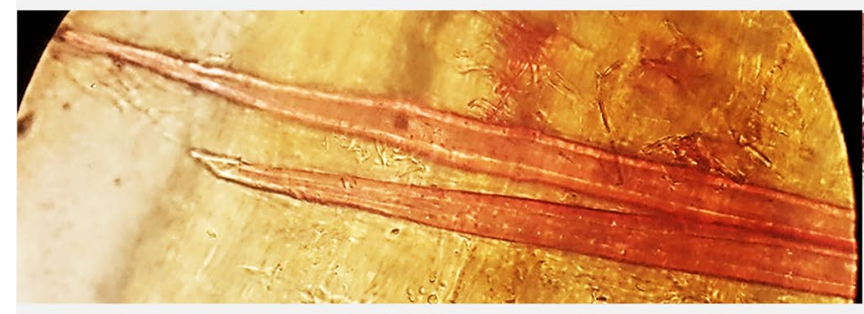

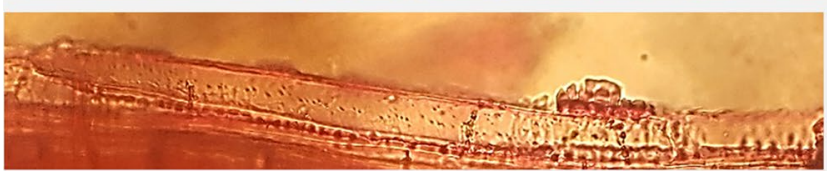

(a)

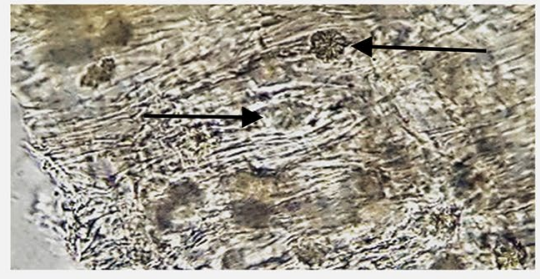

(c)

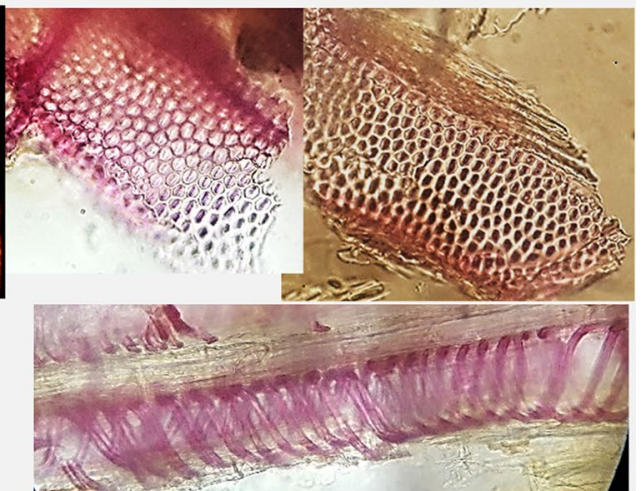

(b)

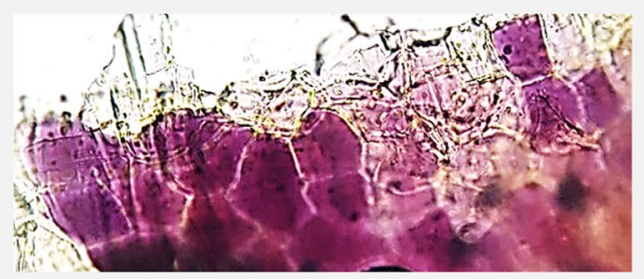

(d)

Fig. 6 Microscopic characteristics of X.strumarium stem powder. a Fibers. b Vessels, pitted, spiral. c Parenchyma cell contains rosette crystals. d Purple pigment

Fig. 7 Microscopic characteristics of X.spinosum leaves powder. a Mesophyll, 10×. b Non-glandular trichome. c Fibers

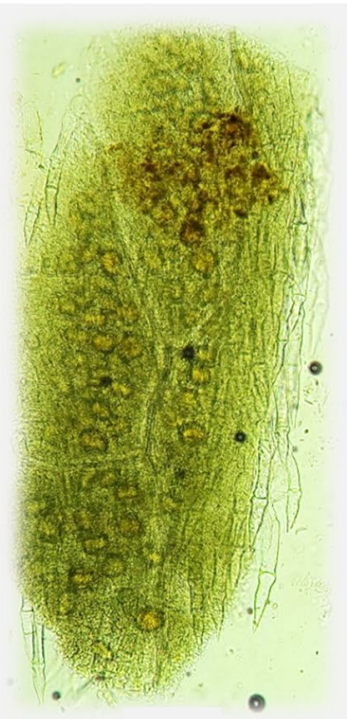

(a)

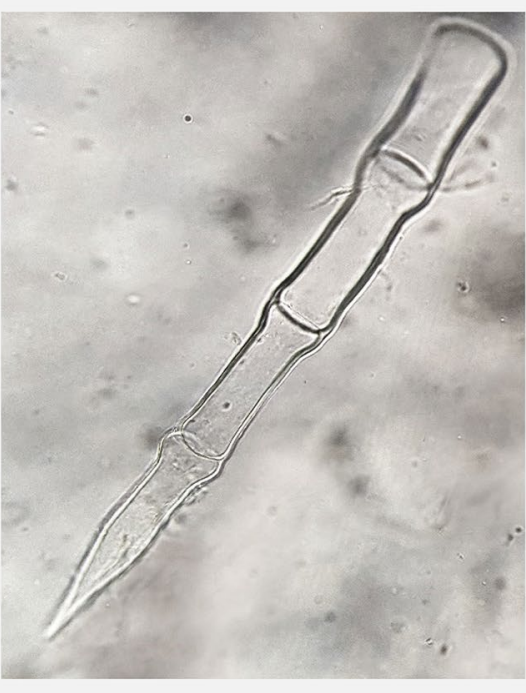

(b)

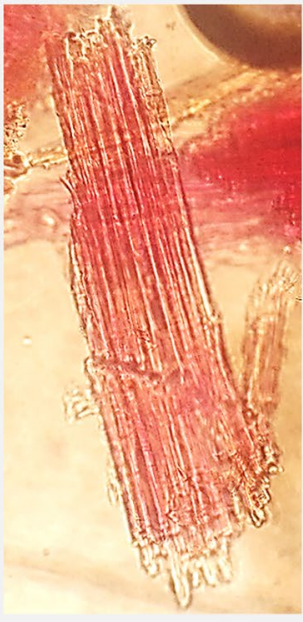

(c) 


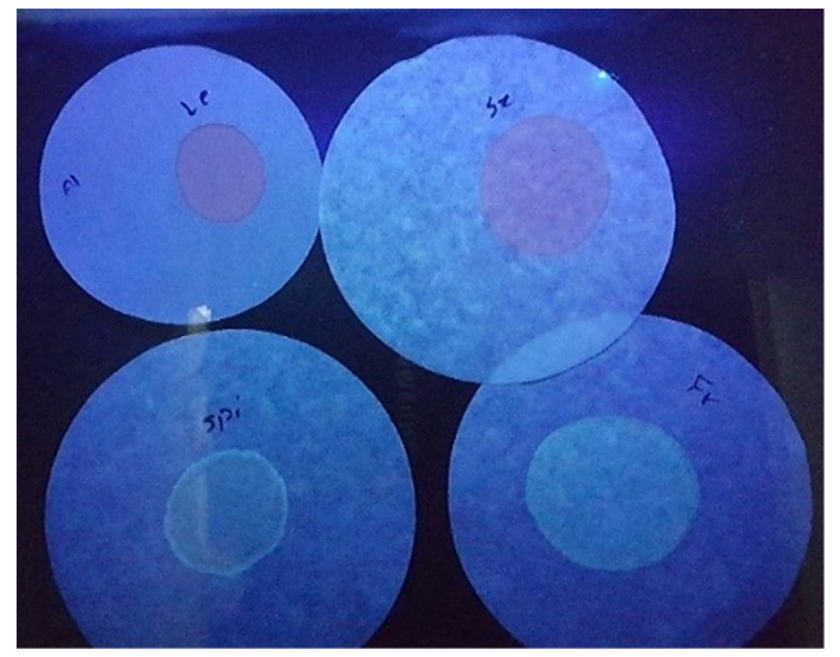

Fig. 8 The color of UV fluorescence of aluminum chloride test

Table 4 Detection of Anthraquinones, Saponins, Tannins

\begin{tabular}{llllll}
\hline Plant part & Le & Se & Fr & Ro & Spi \\
\hline Anthraquinones & - & - & - & - & - \\
Saponins & - & - & - & - & - \\
Tannins & - & - & + & - & + \\
\hline
\end{tabular}

$(+)$ indicates positive detection

$(-)$ indicates negative detection

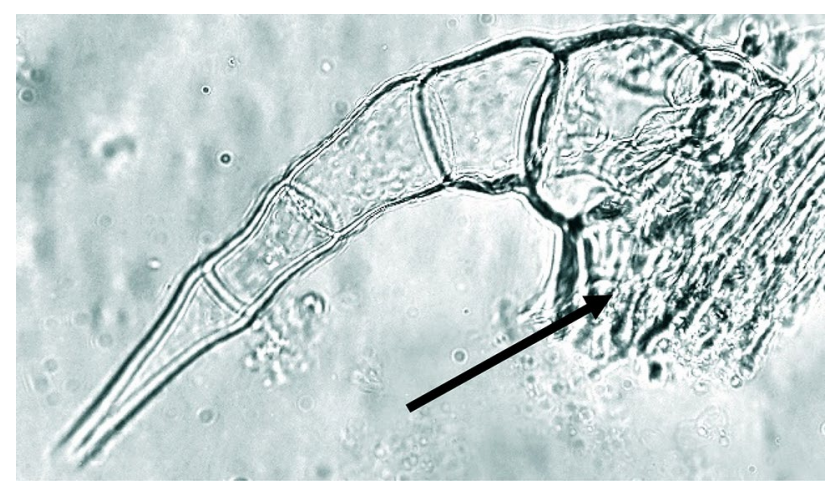

Fig. 9 Presence of the base of the trichome

Mature leaves of $X$. strumarium can be collected before flowering or upon flowering time, so a microscopic examination shows the time when the plant was collected. The flower powder was characterized by fragments of anther endothecium in a large amount, in addition to the distinct pollen grains in their spherical shape or the tricolporate shape. These grains appear freely in large quantities and within the stamens fragment (Fig. 4).

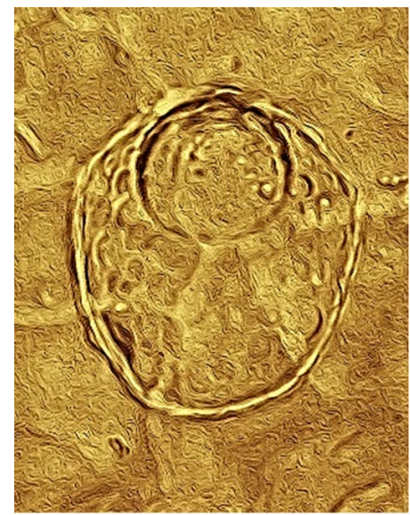

Fig. 10 Broken part of trichome

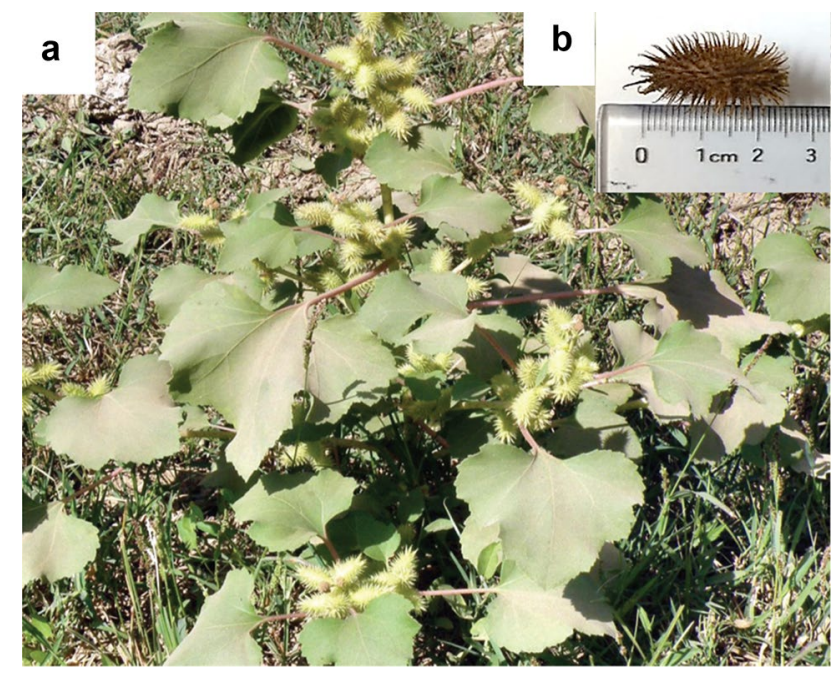

Fig. 11 a X. strumarium, whole plant. b Fruit of X. strumarium

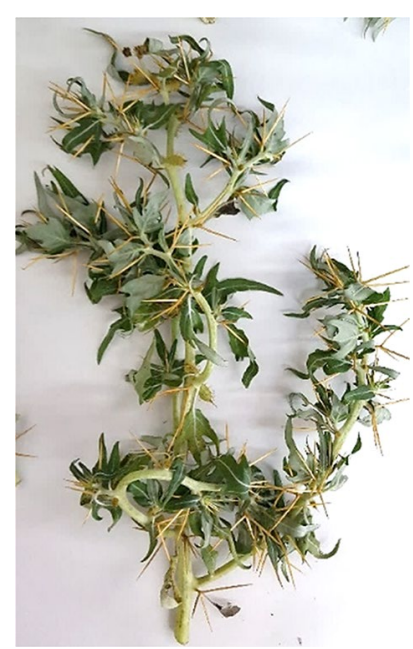

Fig. $12 X$. spinosum 
The stems of $X$. strumarium are less important medically, and it is preferable to separate them from the leaves. Microscopic examination can reveal if stem separation took place during collection or if the leaves were ground with the stem. The appearance of fibers -with dentate walland polygonal cells contained purple pigments in the elements of the leaf powder indicates that they were ground with stems (Fig. 6).

The roots of $X$. strumarium were traditionally used as a bitter tonic and febrifuge [9], so it can be used alone without the leaves, and when the plants were collected in this study, the roots were completely separated from the aerial parts. Therefore, the leaf powder was pure from the elements of roots. The microscopic examination of powdered roots was characterized by the appearance of stone cells scattered within the cortical tissue, in addition to the presence of large pitted vessels (Fig. 3).

The seedling powder can be distinguished by the presence of free and clear separated glandular trichomes within the microscopic examination (Fig. 2) because the number of glandular trichomes is more during this stage of growth and in young leaves [4].

Dharmananda [6] mentioned that the fruits are used in Chinese traditional medicine, but they contain in their spines a toxic substance called a carboxyatractyloside (CAT), and the best way to use fruits safely is to be processed by stir-frying, then the spines are sifted and removed. Only washing or boiling (decocting) the fruits or even frying them without removing the spines is not considered sufficient to get rid of the toxicity. Fruits are used in a dose of 3-10 $\mathrm{g}$, with a safety margin 10-fold compared to the dose causing a significant human reaction (100 g). Microscopic examination of the fruit powder has shown the presence of fusiform fibers and fragments of the mesocarp attached to the endocarp of the fruit (Fig. 5). The collection of the aerial parts of $X$. strumarium at the fruiting time is not an appropriate time to obtain the leaves. If a microscopic examination shows the presence of fruit elements with the leaf elements, this indicates a poor quality of the powder, because the plant was not collected in time and the leaves were mixed with the fruits whose spines should be removed before grinding.

Microscopic examination shows clear differences between the nonglandular trichomes of the powdered leaves of $X$. strumarium and $X$. spinosum (Table 1 ). Clear protuberances were visible on the surface of the trichomes in $X$. strumarium and the basal cells were wider than the apex cells, while nonglandular trichomes of $X$. spinosum were characterized by their smooth surface and all cells were approximately uniform in dimensions.

The morphology of $X$. spinosum is characterized by the presence of spines, and these spines do not have a medical benefit, therefore it is preferable to separate them from the leaves. The microscopy gives a clear indication of the purity of the leaf powder from the spines, as it reveals if the leaves were ground with the spines.

Table 2 shows that the leaves, seedlings, and fruits of X.strumarium contain alkaloids because there are two positive results and this corresponds to Kamboj and Saluja [9] report that the aerial parts contain a mixture of unidentified alkaloids. Farooq et al. [8], Devkota and Das [5], and Malpani et al. [11] also mentioned that the leaves contain alkaloids, while Reeta et al. [14] and Sravani and Lakshmi [15] concluded that the leaves do not contain alkaloids. It appears from the previous table that the leaves of $X$. spinosum do not contain alkaloids.

Table 3 shows that flavonoids are present in the leaves of the two species, but their type is different due to their different fluorescence.

Anthraquinones and saponins detection results were negative, which indicates that all the parts do not contain them, while tannins were found in the fruits of $X$. strumarium and the leaves of $X$. spinosum.

Preliminary tests showed clear differences that can be used to distinguish between the powdered leaves of the two species. In X. strumarium, the detection of alkaloids was positive, the test of aluminum chloride to detect flavonoids gave a brown fluorescence under the UV. The result of testing for tannins was negative. As for $X$. spinosum, the detection of alkaloids was negative, the test of aluminum chloride gave a green fluorescence, and the detection of tannins was positive.

\section{Conclusions}

Microscopic examination returned clear results to reveal the powders of $X$. strumarium parts and the powders of $X$. spinosum leaves. Depending on the photomicrography of the elements, it is possible to control the quality of the powders and reveal the presence of any contamination with powders. Preliminary tests for the leaves of the two species gave an additional method to differentiate the two species of Xanthium. 
Acknowledgements We extend our appreciation to pharmacist Raghad Serri and pharmacist Jamal Darwichah of the Arab International University for their efforts in facilitating the laboratory work, and to Dr. Imad Al-Qadi of the Faculty of Sciences-Damascus University for his assistance in classifying plants.

Author contributions All authors have participated in: conception and design, or analysis and interpretation of the data, drafting the article or revising it critically for important intellectual content, and approval of the final version.

\section{Compliance with ethical standards}

Conflict of interest The author(s) declare that they have no competing interests.

\section{References}

1. Al-Hakim W, Al-Kadi I, Agha E (2012) Atlas of medicinal and aromatic plants. The Arab Center for the Studies of Arid Zones And Dry Lands (ACSAD), Damascus, pp 195-196

2. Jackson BP, Snowdon DW (1990) Atlas of microscopy of medicinal plants, culinary herbs and spices. Belhaven Press, London

3. Bhogaonkar PY, Ahmad SA (2012) Pharmacognostic studies on Xanthium strumarium $\mathrm{L}-\mathrm{a}$ folk unani medicinal herb. Biosci Dis$\operatorname{cov} 3(1): 101-106$

4. Chen F, Hao F, Li C, Gou J, Lu D, Gong F, Tang H, Zhang Y (2013) Identifying three ecological chemotypes of Xanthium strumarium glandular trichomes using a combined NMR and LC-MS method. PLoS ONE 8(10):e76621

5. Devkota A, Das RK (2016) Antifungal activities and phytochemical screening of Xanthium Strumarium. Bio Bull 2(1):121-127

6. Dharmananda S (2003) Safety issues affecting Chinese herbs: the case of Xanthium. Institute for Traditional Medicine (ITM)European Branch. 1: 1-8
7. Evans WC (2009) Trease and Evans' pharmacognosy, Elsevier Health Sciences, 16th edn. Elsevier Limited, Saunders, London

8. Farooq U, Waseem B, Muzaffar R, Tripathi J, Tharani M, Sharma $M$ (2014) A comparative study of phytochemical investigation of Xanthium strumarium medicinal plant. Int J Res Pharm Chem 4(1):96-100

9. Kamboj A, Saluja AK (2010) Phytopharmacological review of Xanthium strumarium L. (Cocklebur). Int J Green Pharm 4:129-139

10. Love D, Dansereau P (1959) Biosystematic studies on Xanthium: Taxonomic appraisal and ecological status. Can J Bot 37:173-208

11. Malpani MO, Rajput PR, Chinchole KV, Kapse SS, Ambarkar KS (2019) Phytochemical screening and antioxidant activity of extracts of Xanthium strumarium, Chrysanthemum and their mixture. Rasayan J Chem 12(4):1901-1908

12. Mouterde (1983) Nouvelle flore du Liban et de la Syrie. Dar Elmachreq Editeurs, Beirut, pp 396-397

13. Munajjed H, Agha E (1997) Phytochemistry and extraction manual. Damascus University, Damascus

14. Reeta S, Kavita G, Arjun P, Rimpal J (2010) Pharmacognostical standardization of leaves of Xanthium strumarium Linn. Phcog J 2(12):492-497

15. Sravani P, Lakshmi SM (2012) Pharmacognostic and phytochemical profiles of Xanthium strumarium L (Asteraceae). Pharm Biol 2(1):20-34

16. The Plant List (2013) Version 1.1. http://www.theplantlist.org/. Accessed 1 Jan 2020

17. Upton R, Graff A, Jolliffe G, Länger R, Williamson E (2011) American herbal pharmacopoeia, botanical pharmacognosy-microscopic characterization of botanical medicines. Taylor \& Francis Group, Milton Park, p 83

18. Weaver SE, Lechowicz MJ (1983) The biology of Canadian weeds. 56. Xanthium strumarium L. Can J Plant Sci 63:211-225

Publisher's Note Springer Nature remains neutral with regard to jurisdictional claims in published maps and institutional affiliations. 